Review Article

\title{
Classification and Characteristics of Mesenchymal Stem Cells and Its Potential Therapeutic Mechanisms and Applications against Ischemic Stroke
}

\author{
Pian Gong, ${ }^{1}$ Wei Zhang, ${ }^{1}$ Yan He, ${ }^{2}$ Jianfeng Wang, ${ }^{1}$ Song $\mathrm{Li}^{3}{ }^{3}$ Songyu Chen, ${ }^{3}$ \\ Qingsong $\mathrm{Ye}\left(\mathbb{0},{ }^{4}\right.$ and Mingchang $\mathrm{Li} \mathbb{1}^{1}$ \\ ${ }^{1}$ Department of Neurosurgery, Renmin Hospital of Wuhan University, Wuhan, Hubei 430060, China \\ ${ }^{2}$ Regenerative Medicine Lab, Tianyou Hospital, Wuhan University of Science and Technology, Wuhan, Hubei 430064, China \\ ${ }^{3}$ Department of Neurosurgery, Shanghai Tenth People's Hospital, Tongji University, Shanghai, China \\ ${ }^{4}$ Center of Regenerative Medicine, Renmin Hospital of Wuhan University, Wuhan, Hubei 430060, China
}

Correspondence should be addressed to Qingsong Ye; qingsongye@hotmail.com and Mingchang Li; mingcli@whu.edu.cn

Received 19 July 2021; Accepted 11 October 2021; Published 9 November 2021

Academic Editor: Jing Yan

Copyright (c) 2021 Pian Gong et al. This is an open access article distributed under the Creative Commons Attribution License, which permits unrestricted use, distribution, and reproduction in any medium, provided the original work is properly cited.

\begin{abstract}
Ischemic stroke is a serious cerebral disease that often induces death and long-term disability. As a currently available therapy for recanalization after ischemic stroke, thrombolysis, including intravenous thrombolysis and endovascular therapy, still cannot be applicable to all patients due to the narrow time window. Mesenchymal stem cell (MSC) transplantation therapy, which can trigger neuronal regeneration and repair, has been considered as a significant advance in treatment of ischemic stroke. MSC transplantation therapy has exhibited its potential to improve the neurological function in ischemic stroke. Our review describes the current progress and future perspective of MSC transplantation therapy in ischemic stroke treatment, including cell types, transplantation approaches, therapeutic mechanisms, and preliminary clinical trials of MSC transplantation, for providing us an update role of MSC transplantation in ischemic stroke treatment.
\end{abstract}

\section{Introduction}

Stroke is the third leading cause of death and disability worldwide that brings a huge burden to the healthcare system [1]. One in six people will suffer from stroke in their lifetime, with over 13.7 million occurring strokes every year and causing 5.8 million people deaths [2]. The major type of stroke is the ischemic stroke, which approximately accounts for 70 percent of all strokes [2]. Although advanced methods for ischemic stroke treatment have been dug up in recent years, no therapy can efficiently improve the overall prognosis of patients [3]. Intravenous administration of recombinant tissue plasminogen activator (rt-PA) thrombolysis is the only drug approved by the Food and Drug Administration to treat the acute ischemic stroke within 4.5 hours [4-6]; however, limited by its therapeutic window, less than $5 \%$ patients benefited [7]. Mechanical thrombectomy, as the alternative treatment for ischemic stroke, entails an intra- arterial (IA) catheter or stent to remove the occluding thrombus, has been proven to be effective within 6 hours of the onset [8]. Both treatments are so highly timedependent; thus, new treatment strategy is imminent. As a new method to treat ischemic stroke, stem cell transplantation was proved to be a hopeful treatment by a growing body of animal experiments and a few successful clinical trials [9, 10]. As the most studied subtype of stem cells, mesenchymal stem cells (MSCs) have been regarded as a promising therapeutic option for ischemic stroke [11]. Therefore, this review is aimed at summarizing the current progress as well as the future perspective of MSC transplantation in ischemic stroke treatment.

\section{Classification and Characteristics of MSCs}

MSCs, also known as mesenchymal stromal cells with properties of self-renewal and multipotential for differentiation, 


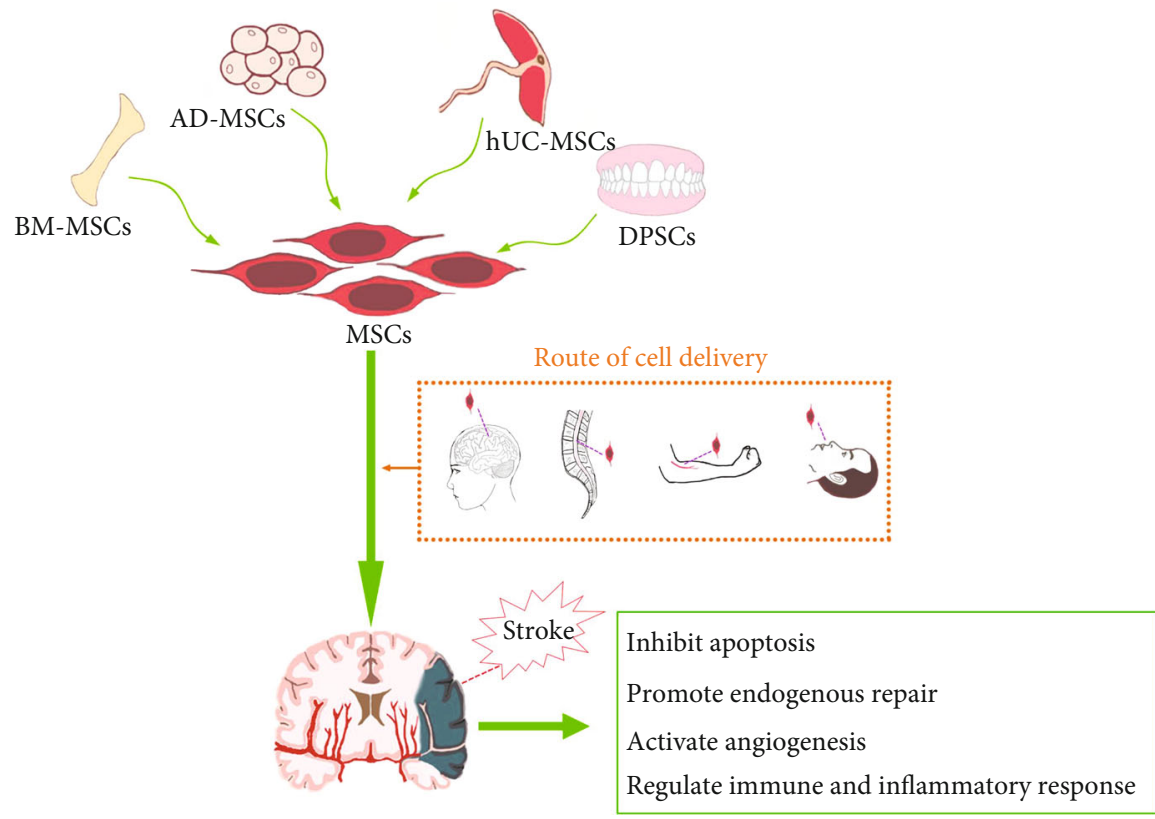

FIgURE 1: Application of MSCs for ischemic stroke. MSCs can be isolated from the bone marrow, adipose tissue, placenta, and teeth and transplanted to the ischemic brain via intracerebral transplantation, intrathecal administration, intravascular administration, and intranasal administration. MSCs can provide neuroprotection and clinical benefits by inhibiting apoptosis, promoting endogenous repair and angiogenesis, and regulating immune and inflammatory response.

can be isolated from various tissues. As shown in Figure 1, varying from cell sources, MSCs can be obtained from the bone marrow, umbilical cord, adipose tissue, placenta, and tissues that originated from the neural crest and others. Furthermore, they can be differed from other stem cells like the hematopoietic stem cells according to the surface molecules expressed by themselves. MSCs express surface molecules such as CD73, CD90, and CD105, and they do not express the surface molecules like CD34, CD45, HLA-antigen D related, CD14 or CD11b, CD79a, or CD19 [12]. MSCs possess multipotential differentiation ability that they can be differentiated into cells like osteoblasts, chondrocytes, and adipocytes. Moreover, they are easy to be isolated and amplified with low immunogenicity and trophic properties $[13,14]$. Unlike embryonic stem cells, the collection, research, and usage of MSCs seldomly raise ethical concerns. Therefore, it is possible to transplanting MSCs to repair multiple injuries.

In the past, researchers obtained MSCs mainly from the bone marrow. MSCs were first found in the bone marrow in 1976 and described as fibroblast-like cells [15]. Later studies revealed that this kind of cells could differentiate into osteogenic, chondrogenic, and adipogenic mesenchymal cell lineages in vitro [16]. Up to date, MSCs in the bone marrow, also known as bone marrow mesenchymal stem cells (BMMSCs), are the most studied and best characterized MSCs. Currently, the most frequent way to obtain BM-MSCs is bone marrow aspiration accompanied by invasion, pain, and the risk of viral and bacterial contamination [17], of which the quality of the obtained cells is determined by age and physical condition of donors [18]. Despite a very low yield, the amplification ability of BM-MSCs is very strong. In the exponential growth period, its doubling time is about 30 to 33 hours. It is reported that human BMMSCs can be propagated in vitro for 40 generations and about 100 million times which can still maintain the stemness [16]. A recent investigation suggested that, in rats underwent acute ischemic stroke, intravenous- (IV-) injected human BM-MSCs can survive and migrate along boundary zones adjacent to the ischemic area and differentiate into the neurons and astrocytes in the microenvironment of the ischemic lesion area, as a result of reduced infarct volume and improved neurological function [19]. Moreover, it is reported that transplanting human BM-MSCs into the infarct area not only stimulates angiogenesis and neurogenesis by secreting multiple cytokines like vascular endothelial growth factor (VEGF), basic fibroblast growth factor (bFGF), and TIMP-3 but also induces differentiation of endogenous stem cells, which results in neuroprotection against ischemic stroke [20, 21]. In addition, transplanted human BM-MSCs were shown to inhibit inflammation and neuronal apoptosis in the ischemic brain of rats [22]. The underlying molecular mechanisms of protective effects induced by BM-MSCs are complex and not entirely clear. Interestingly, a study indicated that, shortly after ischemic stroke in mice, the cell proliferation of BM-MSCs was triggered and promoted, leading to the production of downstream myeloid progenitors and increased presence of inflammatory monocytes and neutrophils, suggesting that BM-MSC could be activated by ischemic injury and that the ischemic injury influenced the primary site of hematopoiesis besides the local inflammation in the ischemic brain [23].

Human umbilical cord mesenchymal stem cells (hUCMSCs) are isolated from the umbilical cord, which are 
featured with the surface molecules of CD29, CD44, CD51, CD105, SH2, and SH3 except CD34 and CD45 [24]. Lots of research explored the effectiveness of hUC-MSCs in ischemic stroke. It was reported that rats underwent middle cerebral artery occlusion (MCAO) for 2 hours and treated with intracerebral hUC-MSC transplantation $1 \mathrm{~d}$ after MCAO operation showed enhancement in neurogenesis and angiogenesis, as a consequence of reduced neurological functional deficits and infarct volume; besides, the hUCMSCs could be detected for at least 5 weeks in the damaged area [25]. The transplanted hUC-MSCs could differentiate into neural progenitors and cells [26], promote the proliferation of neural stem cells and neural differentiation, produce multiple neurotrophic factors, and prevent inflammatory reaction through regulating the activity of the spleen [27], followed by promoted neurological recovery and reduced mortality in animals underwent ischemic stroke. However, hUC-MSC therapy in ischemic stroke is currently limited due to the risk of infection and tumorigenesis [28]. In conclusion, as a kind of MSC that possesses the unique advantage of low immunogenicity without ethical controversy, hUC-MSC is proposed as an excellent candidate in cell therapy for ischemic stroke.

The placenta is thought to be an abundant source that contains two kinds of MSCs: amniotic mesenchymal stromal cells (hA-MSCs) and chorionic mesenchymal stromal cells (hC-MSCs). Both types of cells can be isolated directly from the placenta at the end of gestation through chorionic villus sampling during an invasive prenatal diagnosis. The characteristics of hA-MSCs and hC-MSCs, including low immunogenicity and powerful proliferation $[29,30]$, enable both of them various promising biological properties and make it possible to culture them under good manufacturing practice grade, as a result of numbers of clinical trials on placentaderived stem cell therapy for ischemic stroke [31].

Adipose tissue-derived mesenchymal stem cells (ADMSCs) are abundant, accessible, and easy to obtain using lip aspiration techniques [32]. Efficacy and safety of human AD-MSC in the treatment of stroke has been confirmed in animal models [33]. AD-MSC transplantation was shown to attenuate the neuronal apoptosis and death to exert significant neuroprotective effects through inhibiting the action of $\mathrm{KDM} 6 \mathrm{~B} / \mathrm{BMP} 2 / \mathrm{BMF}$ axis in rats underwent the MCAO [34].

Dental pulp stem cells (DPSCs), originated from the embryonic neural crest and oral-derived epithelial stem cells, are considered as a kind of autologously applicable cells [35]. A report in 2000 first clarified that DPSCs could be isolated and characterized from the third molar [36]. It is currently well known that DPSCs are easily extracted and obtained from human teeth such as deciduous teeth, impacted third molars, and orthodontically extracted premolars. DPSCs have the MSC-like characteristics of high growth capacity and multilineage differentiation potential that they can convert into multiple kinds of cells like neural cells, chondroblasts, and endothelium formative cells. For the advantages of DPSCs, the major one is the easy accessibility without invasive surgical procedures or ethical concerns; another one is that they can maintain their stem cell characteristics after long-term cryopreservation [37, 38]. Moreover, DPSC culture can also be efficiently established from extracted human molars after cryopreservation for up to one month [39].

In addition, DPSCs showed a superior potential for neurogenic differentiation compared with MSCs from other sources like BM-MSCs and AD-MSCs [40]. Upon induction under neuronal differentiation conditions, DPSCs can differentiate into functionally active neuronal cells like mature neurons, dopaminergic-like cells, Schwann cells, and oligodendrocytes [41, 42]. Even without preinduction of neuronal differentiation, DPSCs can express neural stem cell-like markers like nestin and $\beta$-III tubulin [40]. Studies showed that DPSCs can express neurotrophic factors like brainderived neurotrophic factor (BDNF) and VEGF which have been proven to exert neuroprotection against ischemic stroke in both in vitro and in vivo experiments [40]. DPSC-treated primary cortical neurons and astrocytes underwent the oxygen/glucose deprivation (OGD) exhibited promoted neurite regeneration and angiogenesis, and relieved inflammation [19]. DPSC secretion/exosomeimplanted rats with ischemic stroke showed promoted nerve cell proliferation, reduced infarct volume and brain edema, and attenuated neurological dysfunction [43, 44]. Moreover, the culture supernatant of DPSCs that is called dental pulp conditioned medium (DPCM) has been reported to contain cytoprotective factor, revascularization factor, and fibrosis inhibitory factor that contribute to neuronal survival, proliferation, and differentiation [45]; importantly, all of which are well known to be the key mechanisms of neuroprotection against cerebral ischemic stroke. Above evidence shows that both DPSC transplantation and DPCM exert therapeutic effects against ischemic stroke. Furthermore, it was reported that no immune rejection was observed in the brain of mice that had been administered with DPSC implantation derived from rhesus monkeys [46], suggesting that the degree of transplantation rejection of DPSCs was low. When combining with neurotrophic factors, DPSCs can repair both the central nerve and the peripheral nerve once they were attacked by various injuries [47-51]. However, few in-depth studies have examined the effects of DPSCs on the ischemic stroke. Therefore, further research is needed and worthy.

\section{Route of Cell Delivery}

To date, MSCs are delivered via intracerebral transplantation, intrathecal administration, intravascular administration, and intranasal administration to repair ischemic-damaged brain tissue, as shown in Figure 1. Despite intracerebral transplantation and intravascular administration are the commonly used methods [52], there is no optimal route of delivery as every method possesses its own vantages and limitations.

Intracerebral transplantation, also known as stereotactic transplantation, directly injects the MSCs into brain parenchyma or cerebrospinal fluid by stereotactic apparatus. When injected via parenchyma, in order to provide a good microenvironment for stem cells to promote graft survival, 
delivering the MSCs into penumbra or the hemisphere contralateral to the infarct is suggestive $[53,54]$. However, intracerebral transplantation may cause mechanical damage and the number of MSCs is limited. Research has showed that not only the endogenous neural stem cells but also exogenous transplanted MSCs are able to migrate to the ischemic region $[55,56]$. Yet, other researchers argue that even the stem cells successfully arrived at the center of ischemic area, and some survived the initial ischemic damage, the ideal regenerative niches might only appear several days after the stroke in adult mouse brain [57]. Intracerebroventricular injection delivers the MSCs in cerebrospinal fluid mainly to treat brain functional diseases, especially in cerebral ischemia. It was verified that implanted hUC-MSCs by intracerebroventricular injection migrated into the periventricular tissue, followed by promoted functional recovery in the rat model of hypoxic-ischemic encephalopathy [58]. In terms of the timing of administration, both early (12 hours after stroke) and delayed (7 days after stroke) administrations have been proved effective in improving functional outcomes of rats underwent ischemic stroke $[59,60]$. Owing to the importance to control the increased intracranial pressure, multiple administrations of intracerebral transplantation are thought to be impractical, especially for the patients in critical conditions [61]. Intracerebral injection bypasses the blood-brain barrier (BBB) and allows more MSCs into the ischemic lesion. However, as an invasive operation, it is highly technique-sensitive and equipmentdependent [62]. For instance, the positioning accuracy of injection site on MSC transplantation can reach $0.1 \mathrm{~mm}$ using a stereo orientation technique [63].

Intrathecal injection delivers MSCs throughout the entire neuraxis without the invasive brain surgery, making it different from the intraparenchymal and intracerebroventricular administrations [64]. Lim et al. found that therapeutic effects of intrathecal injection of hUC-MSCs can be achieved at a lower dosage in treating cerebral ischemic stroke of rats, compared with intravenous administration [65]. A prospective phase II trial has been initiated in 2019 to assess the effectiveness of allogenic BM-MSC transplantation in severe ischemic stroke, in which eligible patients received BM-MSCs intrathecally at the subacute phase (30 to 90 days following onset) and follow-up assessment were conducted at 7, 30, 90, 180, and 360 days after the injection; after all, the project is in progress with no conclusions published so far [66]. This study may provide a good knowledge of intrathecally implanted BM-MSC therapy for severe ischemic stroke.

Intravascular injection is a safe and feasible delivery way of MSCs, including IV and IA administrations. We find an interesting phenomenon that most clinical trials have used IV injection, and studies with smaller case series preferred IA route [67-69]. Compared with intracerebral injection, intravascular injection is less invasive and allows higher dose and bigger volume of MSCs.

Delivered via IV route, cells are expected to pass the BBB to reach the infarct site of brain and function properly to regenerate new nerve tissue. A research was conducted that the neural stem cells were transplanted into mice at $24 \mathrm{~h}$ after ischemic stroke through IA and IV methods; the results of which showed that IV route leaved the cells traveling through the systemic and pulmonary circulations where cells were more likely to be entrapped in other organs like the spleen, liver, and lungs that $94 \%$ of cells were detected in the lungs at 1 week after stroke, resulting in only a small part of injected portion can reach the brain, and that IA route leaved $69 \%$ of MSCs in the brain several hours after injection and $93 \%$ at 7 days [70]. It was reported that, in rats suffering ischemic stroke, at 14 days after BM-MSC transplantation, the implanted cells through IV injection could not be detected in the ischemic brain and most of them were trapped in the lung, and 35\% of intracerebrally injected MSCs migrated along the corpus callosum to the ischemic region [21]. In the MCAO model of rats, AD-MSCs were injected via IA and IV routes at $24 \mathrm{~h}$ after onset and results showed that at 1-7 days after implantation, the expression level of neurotrophic substance, such as BDNF and VEGF, was increased and level of proapoptotic factors like caspase- 3 and TNF- $\alpha$ was decreased via IA route compared with the IV delivery [71]. IA delivery seems to circumvent the systemic circulation [52], via which the cell number entering the brain was 5 times higher than IV [72]. However, IA injection takes the MSCs nearly 24 hours up to 10 days to reach the brain parenchyma $[73,74]$.

The intranasal route is another less invasive therapeutic option. By passing the BBB, BM-MSCs that were transplanted $24 \mathrm{~h}$ after ischemic stroke in mice could reach the ischemic cortex as early as 1.5 hours postnasal administration, which deposit outside the blood vessels [75]. Cell tracking techniques indicated that the cells can enter the olfactory sheath through the extension adjacent to the olfactory filament after passing through the sieve plate or moving along the surface of the cortex into cerebrospinal fluid and then go into the brain parenchyma $[76,77]$. This route minimizes, if not eliminates, the cell dispersion in systemic circulation to peripheral organs such as the lung. A study showed that the 9-day-old mice administered with MSCs intranasally at 10 days after hypoxic-ischemic brain damage suggested that both of the somatosensory cortex and hippocampus were dramatically regenerated and glial scar around the ischemic site was eliminated at 18 days after the cell therapy [78]. Another study implied that 10-day-old rats administered with hUC-MSCs intranasally at $24 \mathrm{~h}$ posthypoxic-ischemic displayed relieved neuroinflammation and promoted neural regeneration [79].

\section{Treatment Mechanisms of MSCs in Ischemic Stroke}

4.1. MSCs Regulate Immune and Inflammatory Response. Both of immune and inflammatory responses are proven to be significantly involved in the pathogenesis of ischemic stroke. Once the ischemia attacks, the activated innate immunity quickly triggers and promotes the neuroinflammation (Figure 1), as a result of the migration of immune cells from periphery into the ischemic brain [80]. At early stage of ischemic stroke, inflammation limits and relieves the ischemic stress, which is beneficial for the patients. But 


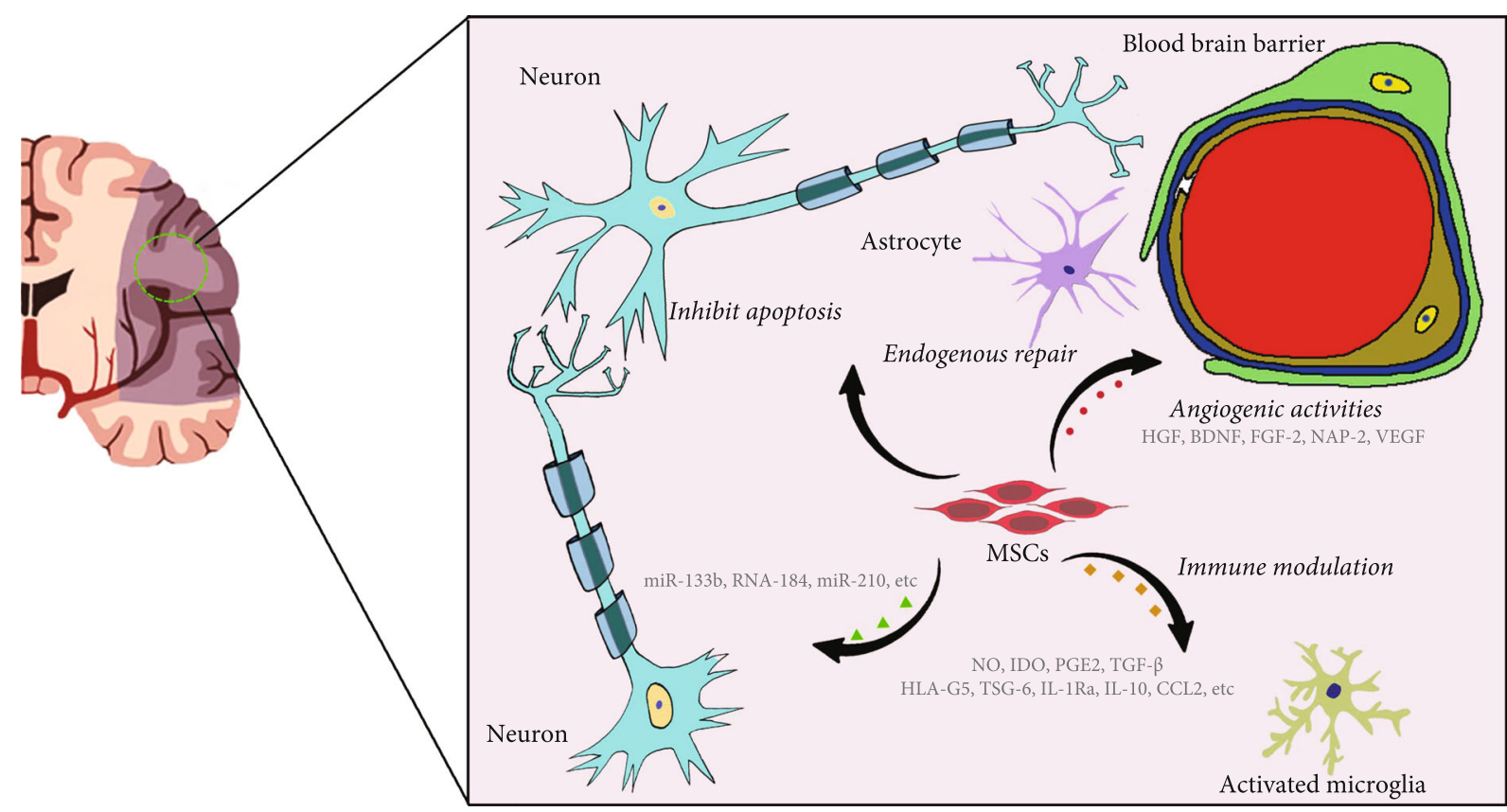

FIGURE 2: Treatment mechanisms of MSCs in ischemic stroke. MSCs produce NO, IDO, PGE2, TGF- $\beta$, HLA-G5, TSG-6, IL-1Ra, IL-10, CCL2, etc., to weaken harmful immune and inflammation responses. MSCs induce activation of microglia and persistent reactive astrogliosis. MSCs interact with microRNAs, like miR-133b, RNA-184, and miR-210, to provide neuroprotective functions. MSCs can secrete biologically active cytokines or factors including BDNF, GDNF, NGF, cCSF, SCF, bFGF, PDGFAA, angiopoietin-2, NAP2, and VEGF to promote angiogenic activities and attenuate blood-brain barrier disruption.

the following uncontrolled inflammation induced by immune cells, for example, the neutrophils, macrophages, NK cells, and $\mathrm{T}$ cells, aggravates the ischemic injury [81, 82]. The ischemic injury is a result of the BBB breakdown, the expression of harmful molecules produced by neural cells, production of glia cell activation-derived proinflammatory factors, and the leukocyte accumulation. MSCs are proven to produce immune regulatory factors such as $\mathrm{NO}$ (in mice), IDO (in human), PGE2, TGF- $\beta$, HLA-G5, TSG6, IL-1Ra, IL-10, and antagonistic variants of CCL2 that weaken harmful immune and inflammation responses and promote tissue repair and regeneration (Figure 2) [83, 84]. The soluble factors derived from MSCs are contributed to the suppression of $\mathrm{T}$ cell proliferation, and the TGF- $\beta$ secreted by MSCs prevents the production of PGE2 and HO-1 and also inhibits the autocrine proliferation of IL-2dependent $\mathrm{T}$ cells (Figure 2). In cerebral ischemia, hUCMSC injection through the tail vein has been shown to modulate TGF- $\beta$, leading to the conversion of naïve $\mathrm{CD} 4^{+}$ $\mathrm{T}$ cells into Th17/Treg and regulation of peripheral immune response, followed by inhibited neuroinflammation and attenuated ischemic injury [85]. In addition, MSCs utilize their property on cell cycle arrest to suppress the IFN$\gamma^{+} / \mathrm{CD}^{+} \mathrm{T}$ cell proliferation through modulating the expression of cyclin D2 and p27kip1, both of which deeply influence the cell cycle of T cells [86]. Moreover, MSCderived doleamine 2,3-dioxygenase (IDO), PGE2, and TGF- $\beta 1$ can downregulate the expression of activated receptors (NKp30, NKp44, and NKG2D), reduce cytotoxicity, inhibit the production of inflammatory cytokines (IFN- $\gamma$ and TNF- $\alpha$ ), and inhibit IL cytotoxic T cell and NK cell pro- liferation (Figure 2) [87, 88]. Early MSC transplantation significantly drives the IL-10 expression, following the decrease in TNF- $\alpha$ production in the ischemic area. BM-MSC transplantations have important roles in attenuating neutrophil infiltration, astrocyte apoptosis, MMP-9 activation, and aquaporin-4 (AQP4) upregulation through suppressing intracellular adhesion molecule 1 (ICAM-1) and activating the p38 signaling pathway, leading to accelerated and enhanced glial scar formation, and reduced BBB disruption and ischemic lesion volume (Figure 2) [89-91].

Splenic inhibition may be another mechanism of MSCs to lighten the immune inflammation. BM-MSC transplantation allows a potential approach for BBB protection in cerebral ischemia that the cell-based therapy attenuates the adverse effect induced by the spleen which increases the BBB permeability and aggravates the BBB disruption [92, 93]. Besides, BM-MSC therapy modulates the peripheral immune response (Figure 1), which is mainly triggered and promoted by the spleen through releasing lymphocytes and proinflammatory factors into the circulatory system at early stage of ischemia $[27,94,95]$.

4.2. MSCs Provide Neurotrophic Functions. At present, the most widely accepted mechanism how MSCs exert the protective effects in ischemic stroke is the neurotrophic factor produced by MSCs through endocrine or paracrine pathway [96]. It has been clarified that the MSCs produce various biologically active cytokines or growth factors like BDNF and $b F G F$ that are crucial for neural regeneration, white matter remodeling, and synaptic plasticity [97-99]. For example, studies have shown that BDNF and bFGF 
expressed by MSCs inhibit the neural death and apoptosis partly through interacting with tyrosine kinase receptors in the animal model of ischemic stroke (Figure 2). As a result, MSC transplantation significantly increased the number of multiple nerve cells synaptophysin, as well as synaptic density, number of myelinated axons, and protuberance growth in the ischemic border area [100]. The increased intensities of oligodendrocyte progenitor cells and mature oligodendrocytes are observed in the border zone of lesion due to BM-MSC transplantation [59]. Moreover, BM-MSC transplantation enhances white matter remodeling through activation of microglia and persistent reactive astrogliosis (Figure 2), leading to the better long-term neurological outcomes [59, 101].

MicroRNAs are strongly linked to MSCs, which play a neuroprotective role in ischemic stroke. MicroRNA profiling analysis revealed that many microRNAs were significantly changed after ischemic stroke $[102,103]$. Xin et al. found that the exosomes from MSCs can modulate the interaction between various microRNAs and neural cells to the structural and functional recovery of neural cells in cerebral ischemia (Figure 2) [104, 105]. It has been proposed that MSCs comodified by targeted peptide and miR-133b can be used as potential therapeutic drugs for cerebral ischemia (Figure 2) [106]. In addition, MSCs act on extracellular vesicles (EVs) in region site to mitigate ischemic injury that the interaction between them promotes neurogenesis and angiogenesis [107]. There is no doubt that MSC treatment has a potential therapeutic value in ischemic stroke with the ability of opening up new avenues and strategies.

4.3. MSCs Induce Angiogenic Activities. Angiogenesis is highly related to the functional recovery of ischemic stroke (Figure 1). In response to the attack of the ischemic stroke, the vascular endothelial cells in the CNS exhibit strong proliferation capability to supply the injured tissue with more oxygen and nutrition. In the next few months after the stroke, as vascular remodeling and vascular density increasing, neuroblasts gradually migrate to the damaged brain area to repair the injured tissue. Existing evidence suggested that MSCs can acquire angiogenic properties through paracrine or autocrine production of appropriate cytokines [108, 109]. Implanted BM-MSCs release many angiogenic growth factors and neurotrophic factors like angiogenin, hepatocyte growth factor, BDNF, and fibroblast growth factor-2 (FGF2 ), insulin-like growth factor-1, neutrophil activating protein-2 (NAP-2), and VEGF (Figure 2) [109]. In addition, some researchers have found that, in the area around the infarction, BM-MSCs facilitate the production of various neuroprotective factors, including stromal cell-derived factor-1 (SDF-1), BDNF, platelet-derived growth factor AA (PDGF-AA), basic fibroblast cell growth factor, angiopoietin-2, CXC chemokine ligand 16, NAP-2, and VEGF receptor-3 (Figure 2) [110]. In rats suffering ischemic stroke, BM-MSC treatment started at $24 \mathrm{~h}$ after onset markedly increases the microvessel density, as a consequence of enhanced angiogenesis in the boundary zone [101, 111]. Zacharek et al. found that coculture of astrocytes with MSCs increased the expression of VEGF and Ang1/Tie2 and signif- icantly increased capillary-like formation of mouse brain endothelial cells [112], resulting in the promotion of the angiogenesis to accelerate tissue repair (Figure 1).

\section{Clinical Trials}

Although preclinical data are promising in terms of both safety and therapeutic efficacy, clinical verification is inevitable for MSCs to treat patients with cerebral ischemia. Up to now, about 1000 clinical trials focused on MSC therapy are currently registered on ClinicalTrials.gov and 20 clinical trials (Table 1) focus on the therapeutic effects of MSCs for cerebral ischemia, among which 4 have been completed and 3 have been withdrawn. A phase II clinical trial from China, in which 10 participants suffering acute ischemic stroke were treated with AD-MSCs via the IV route within 2 weeks after the onset, concluded that AD-MSC implantation was safe and efficient and could improve the neurological function of patients with severe stroke at two years after ischemic stroke [113]. Another 4-year open trial in China involved 18 participants with acute cerebral ischemic stroke showed that participants in the MSC-treated group had fewer serious adverse events compared with the vehicle group and concluded the long-term safety of MSC treatment for acute cerebral ischemic stroke [114]. In a randomized controlled trial with a 2 -year follow-up, 16 patients received the MSC transplantation through the IV route showed improved motor recovery through sensorimotor neuroplasticity, suggesting that MSC treatment was safe and feasible for ischemic stroke [67].

Moreover, clinical trials of 36 patients from Levy et al. [115] showed that the proportion of patients that treated with allogeneic BM-MSCs $\left(1.6 \times 10^{6} / \mathrm{kg}\right)$ via IV route with good functional recovery (Barthel score $\geq 95$ ) increased from $11.4 \%$ of the baseline to $27.3 \%$ at 6 months and $35.5 \%$ at 12 months. Levy et al. concluded that allogeneic BM-MSC injection via IV route was accessible and reasonable in treatment of chronic stroke and suggested behavioral gains in patients with substantial functional defects. In addition, a randomized controlled clinical trial registered as ChiCTRINR-16008908 focused on intrathecal injection of allogeneic BM-MSC four infusions $\left(1 \times 10^{6}\right.$ cells $/ \mathrm{kg}$ body weight $)$ once a week at 1 to 3 months after onset of ischemic stroke is still in progress [66].

\section{Conclusions and Prospects}

At present, MSCs are notably available from multiple sources. Furthermore, they are immunotolerant and hold unequivocal postnatal multilineage potential. MSC transplantation is indeed an excellent therapeutic technique to treat ischemic stroke; however, of which the optimal therapeutic protocols, in terms of MSC subtype, number, preparation, and timing, need to be further studied. Although preclinical studies have shown that MSC therapy occupies with safety and efficacy in the treatment of ischemic stroke, some investigators concern that MSC transplantation may lead to tumor growth, immunodepression, and adverse events of the respiratory system, particularly the pulmonary 


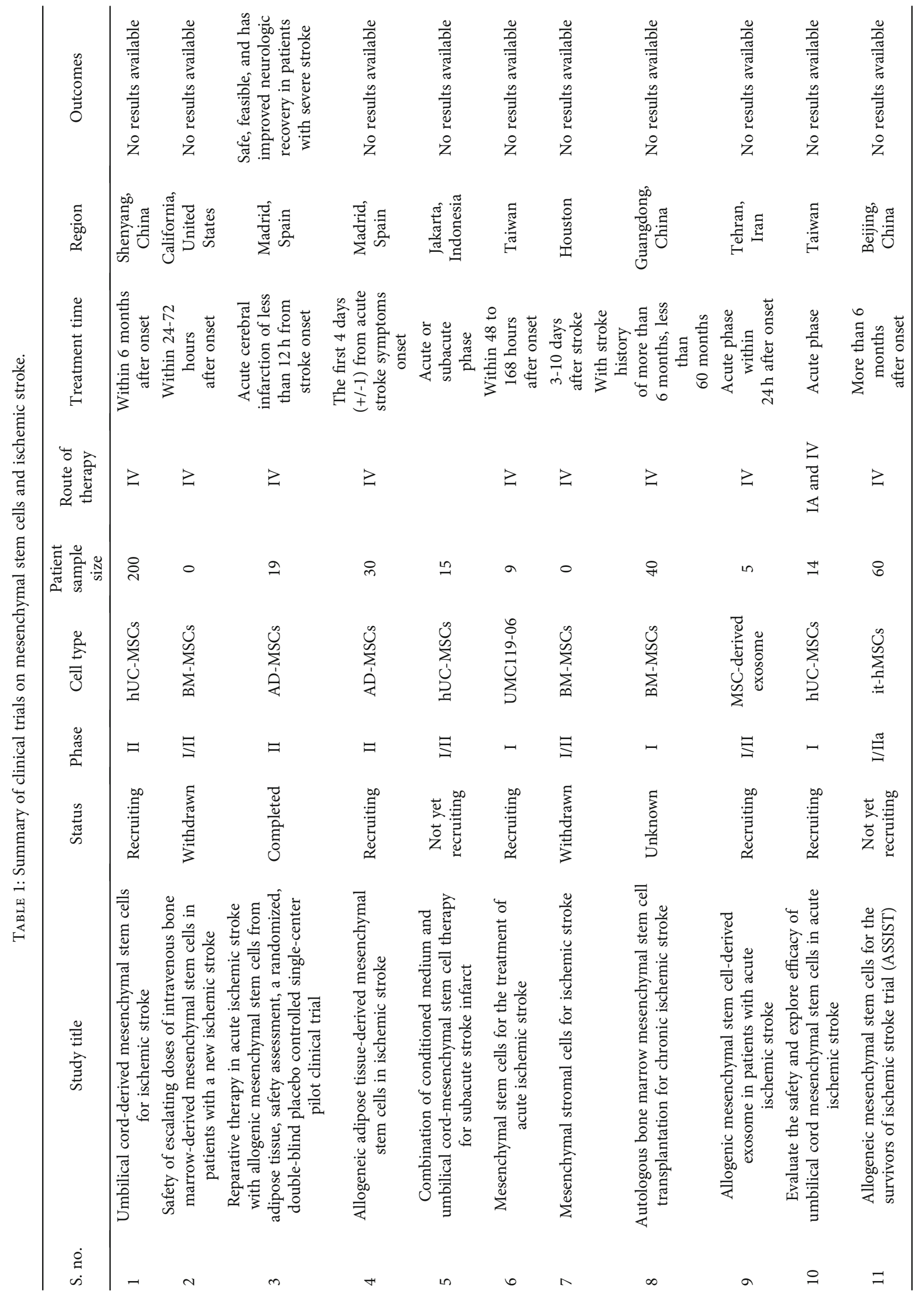




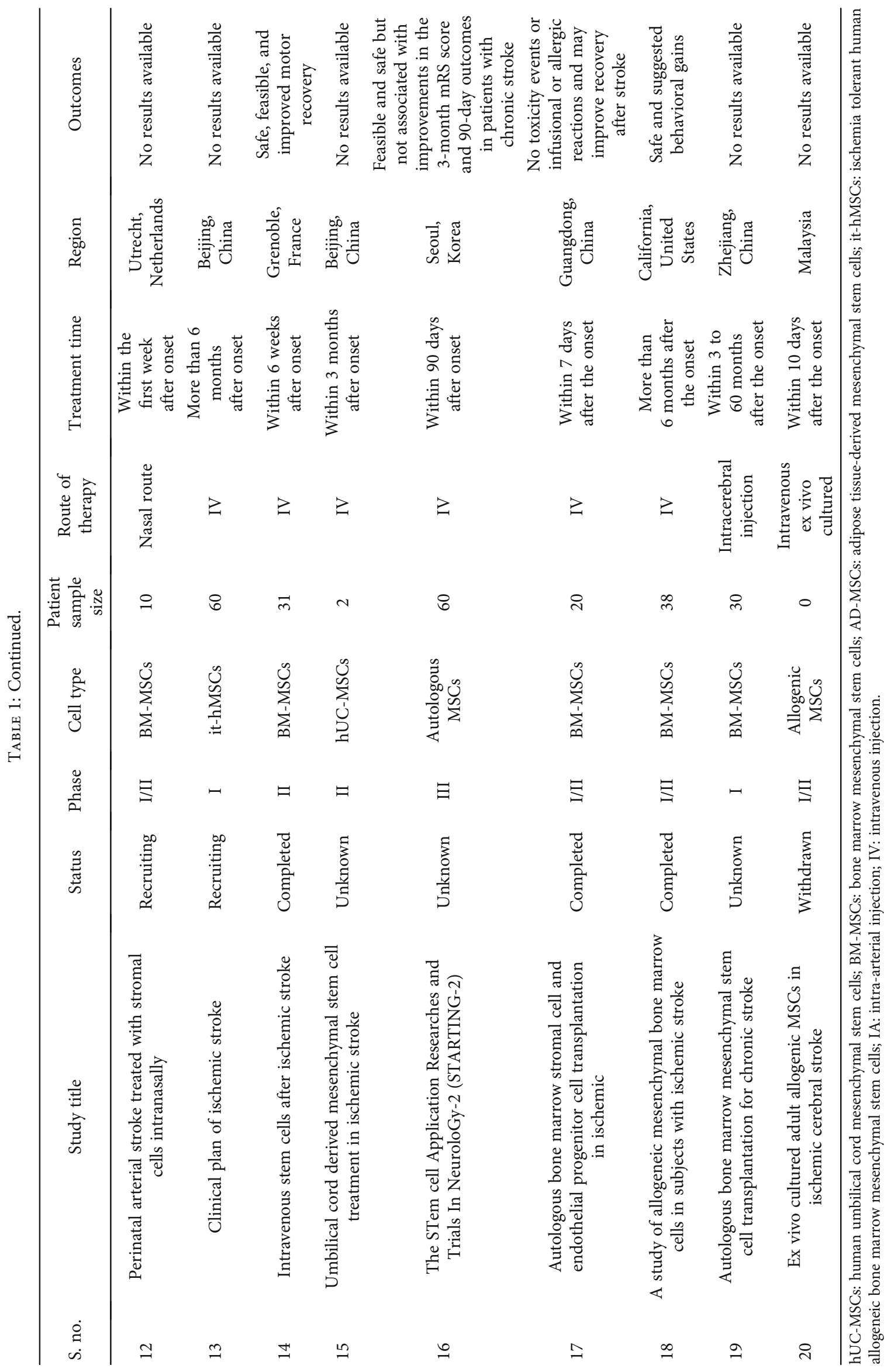


embolism [63]. In addition to the encouraging phase I and phase II data, large-scale phase III clinical trials are required to clear the aforementioned doubts. Hence, we should further conduct not only preclinical studies but also clinical researches to illustrate the effectiveness of MSCs in cerebral ischemia treatment.

\section{Abbreviations}

$\begin{array}{ll}\text { MSCs: } & \text { Mesenchymal stem cells } \\ \text { BM-MSCs: } & \text { Bone marrow mesenchymal stem cells } \\ \text { IV: } & \text { Intravenous } \\ \text { VEGF: } & \text { Vascular endothelial growth factor } \\ \text { bFGF: } & \text { Basic fibroblast growth factor } \\ \text { hUC-MSCs: } & \text { Human umbilical cord mesenchymal stem } \\ & \text { cells } \\ \text { MCAO: } & \text { Middle cerebral artery occlusion } \\ \text { hA-MSCs: } & \text { Human amniotic mesenchymal stromal cells } \\ \text { hC-MSCs: } & \text { Human chorionic mesenchymal stromal cells } \\ \text { AD-MSCs: } & \text { Adipose tissue-derived mesenchymal stem } \\ & \text { cells } \\ \text { DPSCs: } & \text { Dental pulp stem cells } \\ \text { BDNF: } & \text { Brain-derived neurotrophic factor } \\ \text { DPCM: } & \text { Dental pulp conditioned medium } \\ \text { BBB: } & \text { Blood-brain barrier } \\ \text { IA: } & \text { Intra-arterial } \\ \text { NAP-2: } & \text { Neutrophil activating protein-2. }\end{array}$

\section{Conflicts of Interest}

The authors declare that they have no conflicts of interest.

\section{Authors' Contributions}

Pian Gong, Wei Zhang, and Yan He contributed to the conception of this study. Pian Gong, Wei Zhang, Yan He, Jianfeng Wang, Song Li, and Songyu Chen performed the literature research and drafted the manuscript. Qingsong Ye and Mingchang Li participated in revising the paper and finalizing the paper. All authors read and approved the final manuscript. Pian Gong, Wei Zhang, and Yan He contributed equally to this work. Pian Gong, Wei Zhang, and Yan $\mathrm{He}$ are co-first authors.

\section{Acknowledgments}

This study was supported by grants from the National Natural Science Foundation of China (81971870 to M.C.L.).

\section{References}

[1] G B D C o D Collaborators, "Global, regional, and national age-sex-specific mortality for 282 causes of death in 195 countries and territories, 1980-2017: a systematic analysis for the Global Burden of Disease Study 2017," The Lancet, vol. 392, pp. 1736-1788, 2018.

[2] M. S. Phipps and C. A. Cronin, "Management of acute ischemic stroke,” BMJ, vol. 368, p. 16983, 2020.

[3] R. V. Krishnamurthi, V. L. Feigin, M. H. Forouzanfar et al., "Global and regional burden of first-ever ischaemic and hae- morrhagic stroke during 1990-2010: findings from the Global Burden of Disease Study 2010," The Lancet. Global health, vol. 1, pp. e259-e281, 2013.

[4] W. Wang, M. Li, Y. Wang et al., "GSK-3beta inhibitor TWS119 attenuates rtPA-induced hemorrhagic transformation and activates the Wnt/beta-catenin signaling pathway after acute ischemic stroke in rats," Molecular Neurobiology, vol. 53, pp. 7028-7036, 2016.

[5] P. Gong, Z. Zhang, C. Zou et al., "Hippo/YAP signaling pathway mitigates blood-brain barrier disruption after cerebral ischemia/reperfusion injury," Behavioural Brain Research, vol. 356, pp. 8-17, 2019.

[6] P. Gong, Z. Zhang, Y. Zou et al., “Tetramethylpyrazine attenuates blood-brain barrier disruption in ischemia/reperfusion injury through the JAK/STAT signaling pathway," European Journal of Pharmacology, vol. 854, pp. 289-297, 2019.

[7] P. A. Lapchak and J. H. Zhang, "The high cost of stroke and stroke cytoprotection research," Translational Stroke Research, vol. 8, pp. 307-317, 2017.

[8] W. J. Powers, "Acute ischemic stroke," The New England Journal of Medicine, vol. 383, pp. 252-260, 2020.

[9] M. Kawabori, H. Shichinohe, S. Kuroda, and K. Houkin, "Clinical trials of stem cell therapy for cerebral ischemic stroke," International Journal of Molecular Sciences, vol. 21, 2020.

[10] G. Zhou, Y. Wang, S. Gao et al., "Potential mechanisms and perspectives in ischemic stroke treatment using stem cell therapies," Frontiers in Cell and Developmental Biology, vol. 9, article 646927, 2021.

[11] J. W. Chung, W. H. Chang, O. Y. Bang et al., "Efficacy and safety of intravenous mesenchymal stem cells for ischemic stroke," Neurology, vol. 96, pp. e1012-e1023, 2021.

[12] M. Dominici, K. Le Blanc, I. Mueller et al., "Minimal criteria for defining multipotent mesenchymal stromal cells. The International Society for Cellular Therapy position statement," Cytotherapy, vol. 8, pp. 315-317, 2006.

[13] E. A. Kimbrel and R. Lanza, "Next-generation stem cells ushering in a new era of cell-based therapies," Nature Reviews. Drug Discovery, vol. 19, pp. 463-479, 2020.

[14] J. L. Spees, R. H. Lee, and C. A. Gregory, "Mechanisms of mesenchymal stem/stromal cell function," Stem Cell Research \& Therapy, vol. 7, p. 125, 2016.

[15] A. J. Friedenstein, J. F. Gorskaja, and N. N. Kulagina, "Fibroblast precursors in normal and irradiated mouse hematopoietic organs," Experimental Hematology, vol. 4, pp. 267-274, 1976.

[16] M. F. Pittenger, A. M. Mackay, S. C. Beck et al., "Multilineage potential of adult human mesenchymal stem cells," Science, vol. 284, pp. 143-147, 1999.

[17] Y. A. Romanov, V. A. Svintsitskaya, and V. N. Smirnov, "Searching for alternative sources of postnatal human mesenchymal stem cells: candidate MSC-like cells from umbilical cord," Stem Cells, vol. 21, pp. 105-110, 2003.

[18] Y. Zhuo, S. H. Li, M. S. Chen et al., "Aging impairs the angiogenic response to ischemic injury and the activity of implanted cells: combined consequences for cell therapy in older recipients," The Journal of Thoracic and Cardiovascular Surgery, vol. 139, pp. 1286-1294, 2010.

[19] M. Song, J. H. Lee, J. Bae, Y. Bu, and E. C. Kim, "Human dental pulp stem cells are more effective than human bone 
marrow-derived mesenchymal stem cells in cerebral ischemic injury," Cell Transplantation, vol. 26, pp. 1001-1016, 2017.

[20] Z. G. Zhang, L. Zhang, Q. Jiang, and M. Chopp, “Bone marrow-derived endothelial progenitor cells participate in cerebral neovascularization after focal cerebral ischemia in the adult mouse," Circulation Research, vol. 90, pp. 284288, 2002.

[21] Y. Tang, C. Zhang, J. Wang et al., "MRI/SPECT/fluorescent tri-modal probe for evaluating the homing and therapeutic efficacy of transplanted mesenchymal stem cells in a rat ischemic stroke model," Advanced Functional Materials, vol. 25, pp. 1024-1034, 2015.

[22] H. Y. Kim, T. J. Kim, L. Kang et al., "Mesenchymal stem cellderived magnetic extracellular nanovesicles for targeting and treatment of ischemic stroke," Biomaterials, vol. 243, p. 119942, 2020.

[23] G. Courties, F. Herisson, H. B. Sager et al., "Ischemic stroke activates hematopoietic bone marrow stem cells," Circulation Research, vol. 116, pp. 407-417, 2015.

[24] H. S. Wang, S. C. Hung, S. T. Peng et al., "Mesenchymal stem cells in the Wharton's jelly of the human umbilical cord," Stem Cells, vol. 22, pp. 1330-1337, 2004.

[25] C. P. McGuckin, M. Jurga, A. M. Miller et al., "Ischemic brain injury: a consortium analysis of key factors involved in mesenchymal stem cell-mediated inflammatory reduction," Archives of Biochemistry and Biophysics, vol. 534, pp. 88-97, 2013.

[26] M. Messerli, A. Wagner, R. Sager et al., "Stem cells from umbilical cord Wharton's jelly from preterm birth have neuroglial differentiation potential," Reproductive Sciences, vol. 20, pp. 1455-1464, 2013.

[27] J. H. Seo, I. K. Jang, H. Kim et al., "Early immunomodulation by intravenously transplanted mesenchymal stem cells promotes functional recovery in spinal cord injured rats," Cell medicine, vol. 2, pp. 55-67, 2011.

[28] I. Lua, D. James, J. Wang, K. S. Wang, and K. Asahina, "Mesodermal mesenchymal cells give rise to myofibroblasts, but not epithelial cells, in mouse liver injury," Hepatology, vol. 60, pp. 311-322, 2014.

[29] M. H. Abumaree, M. A. Al Jumah, B. Kalionis et al., "Human placental mesenchymal stem cells (pMSCs) play a role as immune suppressive cells by shifting macrophage differentiation from inflammatory M1 to anti-inflammatory M2 macrophages," Stem Cell Reviews and Reports, vol. 9, pp. 620-641, 2013.

[30] J. M. Lee, J. Jung, H. J. Lee et al., "Comparison of immunomodulatory effects of placenta mesenchymal stem cells with bone marrow and adipose mesenchymal stem cells," International Immunopharmacology, vol. 13, pp. 219-224, 2012.

[31] E. Antoniadou and A. L. David, "Placental stem cells," Best Practice \& Research. Clinical Obstetrics \& Gynaecology, vol. 31, pp. 13-29, 2016.

[32] L. Casteilla, V. Planat-Benard, B. Cousin et al., "Plasticity of adipose tissue: a promising therapeutic avenue in the treatment of cardiovascular and blood diseases?," Archives des Maladies du Coeur et des Vaisseaux, vol. 98, pp. 922-926, 2005.

[33] M. Gutierrez-Fernandez, L. Otero-Ortega, J. Ramos-Cejudo, B. Rodriguez-Frutos, B. Fuentes, and E. Diez-Tejedor, "Adipose tissue-derived mesenchymal stem cells as a strategy to improve recovery after stroke," Expert Opinion on Biological Therapy, vol. 15, pp. 873-881, 2015.

[34] Y. Zhang, J. Liu, M. Su, X. Wang, and C. Xie, "Exosomal microRNA-22-3p alleviates cerebral ischemic injury by modulating KDM6B/BMP2/BMF axis," Stem Cell Research \& Therapy, vol. 12, p. 111, 2021.

[35] W. K. Leong, T. L. Henshall, A. Arthur et al., "Human adult dental pulp stem cells enhance poststroke functional recovery through non-neural replacement mechanisms," Stem Cells Translational Medicine, vol. 1, pp. 177-187, 2012.

[36] S. Gronthos, M. Mankani, J. Brahim, P. G. Robey, and S. Shi, "Postnatal human dental pulp stem cells (DPSCs) in vitro and in vivo," Proceedings of the National Academy of Sciences of the United States of America, vol. 97, pp. 13625-13630, 2000.

[37] H. Egusa, W. Sonoyama, M. Nishimura, I. Atsuta, and K. Akiyama, "Stem cells in dentistry-part I: stem cell sources," Journal of Prosthodontic Research, vol. 56, pp. 151-165, 2012.

[38] Y. W. Geng, Z. Zhang, M. Y. Liu, and W. P. Hu, "Differentiation of human dental pulp stem cells into neuronal by resveratrol," Cell Biology International, vol. 41, pp. 1391-1398, 2017.

[39] B. C. Perry, D. Zhou, X. Wu et al., "Collection, cryopreservation, and characterization of human dental pulp-derived mesenchymal stem cells for banking and clinical use," Tissue Engineering. Part C, Methods, vol. 14, pp. 149-156, 2008.

[40] K. Sakai, A. Yamamoto, K. Matsubara et al., "Human dental pulp-derived stem cells promote locomotor recovery after complete transection of the rat spinal cord by multiple neuro-regenerative mechanisms," The Journal of Clinical Investigation, vol. 122, pp. 80-90, 2012.

[41] K. Sanen, W. Martens, M. Georgiou, M. Ameloot, I. Lambrichts, and J. Phillips, "Engineered neural tissue with Schwann cell differentiated human dental pulp stem cells: potential for peripheral nerve repair?," Journal of Tissue Engineering and Regenerative Medicine, vol. 11, pp. 3362-3372, 2017.

[42] B. C. Heng, L. W. Lim, W. Wu, and C. Zhang, "An overview of protocols for the neural induction of dental and oral stem cells in vitro," Tissue Engineering. Part B, Reviews, vol. 22, pp. 220-250, 2016.

[43] S. Li, L. Luo, Y. He et al., "Dental pulp stem cell-derived exosomes alleviate cerebral ischaemia-reperfusion injury through suppressing inflammatory response," Cell Proliferation, vol. 54, article e13093, 2021.

[44] T. Inoue, M. Sugiyama, H. Hattori, H. Wakita, T. Wakabayashi, and M. Ueda, "Stem cells from human exfoliated deciduous tooth-derived conditioned medium enhance recovery of focal cerebral ischemia in rats," Tissue Engineering. Part A, vol. 19, pp. 24-29, 2013.

[45] A. Arthur, S. Shi, A. C. Zannettino, N. Fujii, S. Gronthos, and S. A. Koblar, "Implanted adult human dental pulp stem cells induce endogenous axon guidance," Stem Cells, vol. 27, pp. 2229-2237, 2009.

[46] A. H. Huang, B. R. Snyder, P. H. Cheng, and A. W. Chan, "Putative dental pulp-derived stem/stromal cells promote proliferation and differentiation of endogenous neural cells in the hippocampus of mice," Stem Cells, vol. 26, pp. 26542663, 2008.

[47] L. Luo, A. A. Albashari, X. Wang et al., "Effects of transplanted heparin-poloxamer hydrogel combining dental pulp 
stem cells and bFGF on spinal cord injury repair," Stem Cells International, vol. 2018, Article ID 2398521, 2018.

[48] A. Albashari, Y. He, Y. Zhang et al., "Thermosensitive bFGFmodified hydrogel with dental pulp stem cells on neuroinflammation of spinal cord injury," ACS Omega, vol. 5, pp. 16064-16075, 2020.

[49] P. Wu, Z. Tong, L. Luo et al., "Comprehensive strategy of conduit guidance combined with VEGF producing Schwann cells accelerates peripheral nerve repair," Bioactive Materials, vol. 6, pp. 3515-3527, 2021.

[50] S. Zhu, Y. Ying, Y. He et al., "Hypoxia response elementdirected expression of bFGF in dental pulp stem cells improve the hypoxic environment by targeting pericytes in SCI rats," Bioactive Materials, vol. 6, pp. 2452-2466, 2021.

[51] L. Luo, Y. He, L. Jin et al., "Application of bioactive hydrogels combined with dental pulp stem cells for the repair of large gap peripheral nerve injuries," Bioactive Materials, vol. 6, pp. 638-654, 2021.

[52] L. Wei, Z. Z. Wei, M. Q. Jiang, O. Mohamad, and S. P. Yu, "Stem cell transplantation therapy for multifaceted therapeutic benefits after stroke," Progress in Neurobiology, vol. 157, pp. 49-78, 2017.

[53] M. H. Theus, L. Wei, L. Cui et al., "In vitro hypoxic preconditioning of embryonic stem cells as a strategy of promoting cell survival and functional benefits after transplantation into the ischemic rat brain," Experimental Neurology, vol. 210, pp. 656-670, 2008.

[54] M. Modo, R. P. Stroemer, E. Tang, S. Patel, and H. Hodges, "Effects of implantation site of stem cell grafts on behavioral recovery from stroke damage," Stroke, vol. 33, pp. 2270-2278, 2002.

[55] W. L. Li, S. P. Yu, M. E. Ogle, X. S. Ding, and L. Wei, "Enhanced neurogenesis and cell migration following focal ischemia and peripheral stimulation in mice," Developmental Neurobiology, vol. 68, pp. 1474-1486, 2008.

[56] M. Modo, K. Mellodew, D. Cash et al., "Mapping transplanted stem cell migration after a stroke: a serial, in vivo magnetic resonance imaging study," NeuroImage, vol. 21, pp. 311-317, 2004.

[57] M. Q. Jiang, Y. Y. Zhao, W. Cao et al., "Long-term survival and regeneration of neuronal and vasculature cells inside the core region after ischemic stroke in adult mice," Brain Pathology, vol. 27, pp. 480-498, 2017.

[58] J. Xu, Z. Feng, X. Wang et al., "hUC-MSCs exert a neuroprotective effect via anti-apoptotic mechanisms in a neonatal HIE rat model," Cell Transplantation, vol. 28, pp. 15521559, 2019.

[59] Y. Li, J. Chen, C. L. Zhang et al., "Gliosis and brain remodeling after treatment of stroke in rats with marrow stromal cells," Glia, vol. 49, pp. 407-417, 2005.

[60] S. M. Hosseini, M. Farahmandnia, Z. Razi, S. Delavarifar, and B. Shakibajahromi, " 12 hours after cerebral ischemia is the optimal time for bone marrow mesenchymal stem cell transplantation," Neural Regeneration Research, vol. 10, pp. 904908, 2015.

[61] A. Bakshi, A. L. Barshinger, S. A. Swanger et al., "Lumbar puncture delivery of bone marrow stromal cells in spinal cord contusion: a novel method for minimally invasive cell transplantation," Journal of Neurotrauma, vol. 23, pp. 55-65, 2006.

[62] H. K. Jin, J. E. Carter, G. W. Huntley, and E. H. Schuchman, "Intracerebral transplantation of mesenchymal stem cells into acid sphingomyelinase-deficient mice delays the onset of neurological abnormalities and extends their life span," The Journal of Clinical Investigation, vol. 109, pp. 11831191, 2002.

[63] J. Jiang, Y. Wang, B. Liu, X. Chen, and S. Zhang, "Challenges and research progress of the use of mesenchymal stem cells in the treatment of ischemic stroke," Brain \& Development, vol. 40, pp. 612-626, 2018.

[64] H. Kim, D. L. Na, N. K. Lee, A. R. Kim, S. Lee, and H. Jang, "Intrathecal injection in aA rat model: a potential route to deliver human Wharton"s jelly-derived mesenchymal stem cells into the brain," International Journal of Mmolecular Ssciences, vol. 21, 2020.

[65] J. Y. Lim, C. H. Jeong, J. A. Jun et al., "Therapeutic effects of human umbilical cord blood-derived mesenchymal stem cells after intrathecal administration by lumbar puncture in a rat model of cerebral ischemia," Stem Cell Research \& Therapy, vol. 2, p. 38, 2011.

[66] L. Deng, Q. Peng, H. Wang et al., "Intrathecal injection of allogenic bone marrow-derived mesenchymal stromal cells in treatment of patients with severe ischemic stroke: study protocol for a randomized controlled observer-blinded trial," Translational Stroke Research, vol. 10, pp. 170-177, 2019.

[67] A. Jaillard, M. Hommel, A. Moisan et al., "Autologous mesenchymal stem cells improve motor recovery in subacute ischemic stroke: a randomized clinical trial," Translational Stroke Research, vol. 11, pp. 910-923, 2020.

[68] J. S. Lee, J. M. Hong, G. J. Moon, P. H. Lee, Y. H. Ahn, and O. Y. Bang, "A long-term follow-up study of intravenous autologous mesenchymal stem cell transplantation in patients with ischemic stroke," Stem Cells, vol. 28, pp. 10991106, 2010.

[69] O. Y. Bang, J. S. Lee, P. H. Lee, and G. Lee, "Autologous mesenchymal stem cell transplantation in stroke patients," Annals of Neurology, vol. 57, pp. 874-882, 2005.

[70] A. V. Pendharkar, J. Y. Chua, R. H. Andres et al., "Biodistribution of neural stem cells after intravascular therapy for hypoxic-ischemia," Stroke, vol. 41, pp. 2064-2070, 2010.

[71] G. Du, Y. Liu, M. Dang et al., "Comparison of administration routes for adipose-derived stem cells in the treatment of middle cerebral artery occlusion in rats," Acta Histochemica, vol. 116, pp. 1075-1084, 2014.

[72] J. Biernaskie, D. Corbett, J. Peeling, J. Wells, and H. Lei, “A serial MR study of cerebral blood flow changes and lesion development following endothelin-1-induced ischemia in rats," Magnetic Resonance in Medicine, vol. 46, pp. 827-830, 2001.

[73] J. M. Karp and G. S. Leng Teo, "Mesenchymal stem cell homing: the devil is in the details," Cell Stem Cell, vol. 4, pp. 206216, 2009.

[74] M. T. Harting, F. Jimenez, H. Xue et al., "Intravenous mesenchymal stem cell therapy for traumatic brain injury," Journal of Neurosurgery, vol. 110, pp. 1189-1197, 2009.

[75] N. Wei, S. P. Yu, X. Gu et al., "Delayed intranasal delivery of hypoxic-preconditioned bone marrow mesenchymal stem cells enhanced cell homing and therapeutic benefits after ischemic stroke in mice," Cell Transplantation, vol. 22, pp. 977-991, 2013.

[76] L. Danielyan, R. Schafer, A. von Ameln-Mayerhofer et al., "Intranasal delivery of cells to the brain," European Journal of Cell Biology, vol. 88, pp. 315-324, 2009. 
[77] C. Galeano, Z. Qiu, A. Mishra et al., "The route by which intranasally delivered stem cells enter the central nervous system," Cell Transplantation, vol. 27, pp. 501-514, 2018.

[78] V. Donega, C. H. Nijboer, G. van Tilborg, R. M. Dijkhuizen, A. Kavelaars, and C. J. Heijnen, "Intranasally administered mesenchymal stem cells promote a regenerative niche for repair of neonatal ischemic brain injury," Experimental Neurology, vol. 261, pp. 53-64, 2014.

[79] C. A. McDonald, Z. Djuliannisaa, M. Petraki et al., "Intranasal delivery of mesenchymal stromal cells protects against neonatal hypoxic(-)ischemic brain injury," International Journal of Molecular Sciences, vol. 20, 2019.

[80] Y. Shi, R. K. Leak, R. F. Keep, and J. Chen, "Translational stroke research on blood-brain barrier damage: challenges, perspectives, and goals," Translational Stroke Research, vol. 7, pp. 89-92, 2016.

[81] D. Petrovic-Djergovic, S. N. Goonewardena, and D. J. Pinsky, "Inflammatory disequilibrium in stroke," Circulation Research, vol. 119, pp. 142-158, 2016.

[82] T. Shichita, M. Ito, and A. Yoshimura, "Post-ischemic inflammation regulates neural damage and protection," Frontiers in Cellular Neuroscience, vol. 8, p. 319, 2014.

[83] Y. Wang, X. Chen, W. Cao, and Y. Shi, "Plasticity of mesenchymal stem cells in immunomodulation: pathological and therapeutic implications," Nature Immunology, vol. 15, pp. 1009-1016, 2014.

[84] C. R. Harrell, M. G. Jankovic, C. Fellabaum et al., "Molecular mechanisms responsible for anti-inflammatory and immunosuppressive effects of mesenchymal stem cell-derived factors," Advances in Experimental Medicine and Biology, vol. 1084, pp. 187-206, 2019.

[85] Q. Cheng, Z. Zhang, S. Zhang et al., "Human umbilical cord mesenchymal stem cells protect against ischemic brain injury in mouse by regulating peripheral immunoinflammation," Brain Research, vol. 1594, pp. 293-304, 2015.

[86] S. Glennie, I. Soeiro, P. J. Dyson, E. W. Lam, and F. Dazzi, "Bone marrow mesenchymal stem cells induce division arrest anergy of activated T cells," Blood, vol. 105, pp. 2821-2827, 2005.

[87] M. Li, X. Sun, X. Kuang, Y. Liao, H. Li, and D. Luo, "Mesenchymal stem cells suppress CD8+ T cell-mediated activation by suppressing natural killer group 2 , member $\mathrm{D}$ protein receptor expression and secretion of prostaglandin E2, indoleamine 2, 3-dioxygenase and transforming growth factorbeta," Clinical and Experimental Immunology, vol. 178, pp. 516-524, 2014.

[88] G. M. Spaggiari, H. Abdelrazik, F. Becchetti, and L. Moretta, "MSCs inhibit monocyte-derived DC maturation and function by selectively interfering with the generation of immature DCs: central role of MSC-derived prostaglandin E2," Blood, vol. 113, pp. 6576-6583, 2009.

[89] Z. Cheng, L. Wang, M. Qu et al., "Mesenchymal stem cells attenuate blood-brain barrier leakage after cerebral ischemia in mice," Journal of Neuroinflammation, vol. 15, p. $135,2018$.

[90] G. Tang, Y. Liu, Z. Zhang et al., "Mesenchymal stem cells maintain blood-brain barrier integrity by inhibiting aquaporin-4 upregulation after cerebral ischemia," Stem Cells, vol. 32, pp. 3150-3162, 2014.

[91] N. Pavlichenko, I. Sokolova, S. Vijde et al., "Mesenchymal stem cells transplantation could be beneficial for treatment of experimental ischemic stroke in rats," Brain Research, vol. 1233, pp. 203-213, 2008.

[92] P. A. Walker, S. K. Shah, F. Jimenez et al., "Intravenous multipotent adult progenitor cell therapy for traumatic brain injury: preserving the blood brain barrier via an interaction with splenocytes," Experimental Neurology, vol. 225, pp. 341-352, 2010.

[93] T. A. Womble, S. Green, M. Shahaduzzaman et al., "Monocytes are essential for the neuroprotective effect of human cord blood cells following middle cerebral artery occlusion in rat," Molecular and Cellular Neurosciences, vol. 59, pp. 76-84, 2014.

[94] H. Offner, S. Subramanian, S. M. Parker, M. E. Afentoulis, A. A. Vandenbark, and P. D. Hurn, "Experimental stroke induces massive, rapid activation of the peripheral immune system," Journal of Cerebral Blood Flow and Metabolism: Official Journal of the International Society of Cerebral Blood Flow and Metabolism, vol. 26, pp. 654-665, 2006.

[95] C. C. Leonardo, A. A. Hall, L. A. Collier, C. T. Ajmo Jr., A. E. Willing, and K. R. Pennypacker, "Human umbilical cord blood cell therapy blocks the morphological change and recruitment of CD11b-expressing, isolectin-binding proinflammatory cells after middle cerebral artery occlusion," Journal of Neuroscience Research, vol. 88, pp. 1213-1222, 2010.

[96] R. Das, H. Jahr, G. J. van Osch, and E. Farrell, “The role of hypoxia in bone marrow-derived mesenchymal stem cells: considerations for regenerative medicine approaches," Tissue Engineering Part B, Reviews, vol. 16, pp. 159-168, 2010.

[97] X. Chen, Y. Li, L. Wang et al., "Ischemic rat brain extracts induce human marrow stromal cell growth factor production," Neuropathology, vol. 22, pp. 275-279, 2002.

[98] J. Alder, B. C. Kramer, C. Hoskin, and S. Thakker-Varia, "Brain-derived neurotrophic factor produced by human umbilical tissue-derived cells is required for its effect on hippocampal dendritic differentiation," Developmental Neurobiology, vol. 72, pp. 755-765, 2012.

[99] C. A. Ribeiro, J. S. Fraga, M. Graos et al., "The secretome of stem cells isolated from the adipose tissue and Wharton jelly acts differently on central nervous system derived cell populations," Stem Cell Research \& Therapy, vol. 3, p. 18, 2012.

[100] X. Ding, Y. Li, Z. Liu et al., "The sonic hedgehog pathway mediates brain plasticity and subsequent functional recovery after bone marrow stromal cell treatment of stroke in mice," Journal of Cerebral Blood Flow and Metabolism: Official Journal of the International Society of Cerebral Blood Flow and Metabolism, vol. 33, pp. 1015-1024, 2013.

[101] M. Yang, X. Wei, J. Li, L. A. Heine, R. Rosenwasser, and L. Iacovitti, "Changes in host blood factors and brain glia accompanying the functional recovery after systemic administration of bone marrow stem cells in ischemic stroke rats," Cell Transplantation, vol. 19, pp. 1073-1084, 2010.

[102] S. P. Liu, R. H. Fu, H. H. Yu et al., "MicroRNAs regulation modulated self-renewal and lineage differentiation of stem cells," Cell Transplantation, vol. 18, pp. 1039-1045, 2009.

[103] Z. Zhang, J. Yang, W. Yan, Y. Li, Z. Shen, and T. Asahara, "Pretreatment of cardiac stem cells with exosomes derived from mesenchymal stem cells enhances myocardial repair," Journal of the American Heart Association, vol. 25, article e002856, 2016.

[104] H. Xin, Y. Li, Z. Liu et al., "miR-133b promotes neural plasticity and functional recovery after treatment of stroke with 
multipotent mesenchymal stromal cells in rats via transfer of exosome-enriched extracellular particles," Stem Cells, vol. 31, pp. 2737-2746, 2013.

[105] H. Xin, F. Wang, Y. Li et al., "Secondary release of exosomes from astrocytes contributes to the increase in neural plasticity and improvement of functional recovery after stroke in rats treated with exosomes harvested from microRNA 133boverexpressing multipotent mesenchymal stromal cells," Cell Transplantation, vol. 26, pp. 243-257, 2017.

[106] B. Huang, X. C. Jiang, T. Y. Zhang et al., "Peptide modified mesenchymal stem cells as targeting delivery system transfected with miR-133b for the treatment of cerebral ischemia," International Journal of Pharmaceutics, vol. 531, pp. 90-100, 2017.

[107] G. J. Moon, J. H. Sung, D. H. Kim et al., “Application of mesenchymal stem cell-derived extracellular vesicles for stroke: biodistribution and microRNA study," Translational Stroke Research, vol. 10, pp. 509-521, 2019.

[108] C. J. Cunningham, E. Redondo-Castro, and S. M. Allan, “The therapeutic potential of the mesenchymal stem cell secretome in ischaemic stroke," Journal of Cerebral Blood Flow and Metabolism: Official Journal of the International Society of Cerebral Blood Flow and Metabolism, vol. 38, pp. 12761292, 2018.

[109] A. Bronckaers, P. Hilkens, W. Martens et al., "Mesenchymal stem/stromal cells as a pharmacological and therapeutic approach to accelerate angiogenesis," Pharmacology \& Therapeutics, vol. 143, pp. 181-196, 2014.

[110] Y. C. Lin, T. L. Ko, Y. H. Shih et al., "Human umbilical mesenchymal stem cells promote recovery after ischemic stroke," Stroke, vol. 42, pp. 2045-2053, 2011.

[111] M. Dao, C. C. Tate, M. McGrogan, and C. C. Case, "Comparing the angiogenic potency of naive marrow stromal cells and Notch-transfected marrow stromal cells," Journal of Translational Medicine, vol. 11, p. 81, 2013.

[112] A. Zacharek, J. Chen, X. Cui et al., "Angiopoietin1/Tie2 and VEGF/Flk1 induced by MSC treatment amplifies angiogenesis and vascular stabilization after stroke," Journal of Cerebral Blood Flow and Metabolism: Official Journal of the International Society of Cerebral Blood Flow and Metabolism, vol. 27, pp. 1684-1691, 2007.

[113] E. Diez-Tejedor, M. Gutierrez-Fernandez, P. MartinezSanchez et al., "Reparative therapy for acute ischemic stroke with allogeneic mesenchymal stem cells from adipose tissue: a safety assessment: a phase II randomized, double-blind, placebo-controlled, single-center, pilot clinical trial," Journal of Stroke and Cerebrovascular Diseases, vol. 23, pp. 2694-2700, 2014.

[114] J. Fang, Y. Guo, S. Tan et al., “Autologous endothelial progenitor cells transplantation for acute ischemic stroke: a 4-year follow-up study," Stem Cells Translational Medicine, vol. 8, pp. 14-21, 2019.

[115] M. L. Levy, J. R. Crawford, N. Dib, L. Verkh, N. Tankovich, and S. C. Cramer, "Phase I/II study of safety and preliminary efficacy of intravenous allogeneic mesenchymal stem cells in chronic stroke," Stroke, vol. 50, pp. 2835-2841, 2019. 